\title{
Peripheral Motor Neuropathy, CTCAE
}

National Cancer Institute

\section{Source}

National Cancer Institute. Peripheral Motor Neuropathy, CT CAE. NCI Thesaurus. Code C143750.

A disorder characterized by damage or dysfunction of the peripheral motor nerves. 